POS $\quad$ PROCEEDINGS

\title{
Nucleosynthesis Reactions with the High-Intensity SARAF-LiLiT Neutron Source
}

\section{Moshe Tessler, Michael Paul ${ }^{1}$, Tala Palchan}

The Hebrew University, Jerusalem, Israel

E-mail:paul@vms.huji.ac.il

Shlomi Halfon, Leonid Weissman, Nir Hazenshprung, Arik Kreisel, Tzach Makmal, Asher Shor, Ido Silverman

Soreq Nuclear Research Center, Yavne

Melina Avila Coronado, S. Almaraz-Calderon, Wei Jiang, Zheng-Tian Lu, Peter Mueller, Richard Pardo, K. Ernst Rehm, Robert Scott, Rashi Talwar, Claudio Ugalde, Richard Vondrasek, Jake Zappala

Argonne National Laboratory, Argonne, IL, USA

\section{Daniel Santiago-Gonzalez}

Dpt. of Physics and Astronomy, Louisiana State University, Baton Rouge, LA, US and Argonne National Laboratory, Argonne, IL, USA

Philippe Collon, Yoav Kashiv

University of Notre Dame, Notre Dame, IN, USA

Mario Weigand, Tanja Heftrich, René Reifarth, Daniel Veltum

Goethe University of Frankfurt, Frankfurt, Germany

Roland Purtschert

University of Bern, Bern, Switzerland

Carlos Guerrero, Jorge Lerendegui Marco, Jose Manuel Quesada

University of Seville, Seville, Spain

Ulli Köster

Institut Laue-Langevin, Grenoble, France

Dorothea Schumann, Rugard Dressler, Stephan Heinitz, Niko Kivel, Emilio Andrea Maugeri

Paul Scherrer Institute, Villigen, Switzerland

We present a status report of recent neutron capture experiments performed with the $\mathrm{mA}$-proton beam (1.92 MeV, $3 \mathrm{~kW}$ ) of the Soreq Applied Research Accelerator Facility (SARAF) and the Liquid-Lithium Target (LiLiT). Experiments and preliminary results for $(\mathrm{n}, \gamma)$ reactions on ${ }^{36,38} \mathrm{Ar}$, studied for the first time with 30-keV neutrons, on ${ }^{\text {nat }} \mathrm{Kr}$, ${ }^{\text {nat }} \mathrm{Ce}$ and on radioactive targets ${ }^{147} \mathrm{Pm}$ and ${ }^{171} \mathrm{Tm}$ are described.

Australia

${ }^{1}$ Speaker 


\section{Introduction}

Neutron-induced reactions remain at the forefront of experimental investigations for the understanding of stellar nucleosynthesis and chemical evolution of the Galaxy. We report on recent experiments performed with the mA-proton beam at $1.92 \mathrm{MeV}(2-3 \mathrm{~kW})$ from the Soreq Applied Research Accelerator Facility (SARAF) and the Liquid-Lithium Target (LiLiT), yielding high-intensity $\left(3-5 \times 10^{10} \mathrm{n} / \mathrm{s}\right)$ quasi-Maxwellian neutrons at $\sim 30 \mathrm{keV}[1,2]$, close to the thermal conditions of the stellar slow (s-) neutron capture process. First experiments were dedicated to benchmark the experimental system by measuring the Maxwellian Averaged Cross Section (MACS) of several targets. The MACS of ${ }^{94} \mathrm{Zr}$ and ${ }^{96} \mathrm{Zr}$, important isotopes for understanding the $s$-process evolution, were determined as $28.0 \pm 0.6 \mathrm{mb}$ and $12.4 \pm 0.5 \mathrm{mb}$ respectively [3], based on activation measurements and detailed analysis, in good agreement with previous measurements, with lower uncertainties. Interestingly, it was realized [3] that, in addition to the neutron field mentioned above, the SARAF-LiLiT setup yields high-energy $(17.6$ and $14.6 \mathrm{MeV})$ $\gamma$-rays due to the ${ }^{7} \operatorname{Li}(\mathrm{p}, \gamma)$ proton capture with considerable intensities $\left(\sim 3 \times 10^{8}\right.$ and $\sim 4 \times 10^{8} \gamma \mathrm{s}^{-1}$ $\mathrm{mA}^{-1}$, respectively) and produces $(\gamma, \mathrm{n})$ activation products. The latter reactions can be studied separately with a proton beam at an energy below the ${ }^{7} \mathrm{Li}(\mathrm{p}, \mathrm{n})$ neutron threshold.

Using $\alpha-, \beta-, \gamma$-spectrometry and atom-counting techniques (accelerator mass spectrometry, atom-trap trace analysis), we are extending our experimental studies of neutron capture reactions (Table 1) to several targets of astrophysical interest: (i) ${ }^{36,38} \operatorname{Ar}(\mathrm{n}, \gamma)$ reactions investigated for the first time with 30-keV neutrons; (ii) isotopes of $\mathrm{Ga}$, Se and $\mathrm{Kr}$, important for the weak s-process; (iii) isotopes of $\mathrm{Xe}$ and $\mathrm{Ce}$, important for the main s-process; (iv) ${ }^{208} \mathrm{~Pb}$ and ${ }^{209} \mathrm{Bi}$, at the end of the s-process path. The high neutron intensity enables MACS measurements of low-abundance or radioactive targets; $(\mathrm{n}, \gamma)$ reactions on s-process branching points ${ }^{147} \mathrm{Pm},{ }^{171} \mathrm{Tm}$ are investigated.

Table 1: $(n, \gamma)$ and $(\gamma, n)$ reactions under investigation at SARAF-LiLiT

\begin{tabular}{|c|c|c|c|}
\hline Reaction & Detection tech. & $\begin{array}{c}\text { Hebrew U, SARAF and } \\
\text { collaborations below }\end{array}$ & Ref./status \\
\hline $94,96 \mathrm{Zr}(\mathrm{n}, \gamma)$ & $\gamma$ spec. & - & [3] \\
\hline${ }^{90} \operatorname{Zr}(\gamma, \mathrm{n})$ & $\gamma$ spec. & - & [3] \\
\hline${ }^{23} \mathrm{Na},{ }^{35,37} \mathrm{Cl}(\mathrm{n}, \gamma)$ & $\gamma$ spec., AMS & ANU,Goethe U,Rossendorf & prelim, [4] \\
\hline${ }^{36,38} \operatorname{Ar}(\mathrm{n}, \gamma)$ & AMS,LLC & ANL, Goethe U, U Bern & prelim,this conf. \\
\hline${ }^{69,71} \mathrm{Ga}(\mathrm{n}, \gamma)$ & $\gamma$ spec. & - & in progress \\
\hline $74,78,80,82 \mathrm{Se}(\mathrm{n}, \gamma)$ & $\gamma$ spec. & - & in progress \\
\hline $78,80,84,86 \operatorname{Kr}(\mathrm{n}, \gamma)$ & $\gamma$, ATTA,LLC & ANL, Goethe U, U Bern & prelim,this conf. \\
\hline${ }^{80,82,86} \operatorname{Kr}(\gamma, \mathrm{n})$ & $\gamma$, ATTA & ANL, Goethe U & in progress \\
\hline${ }^{92} \operatorname{Zr}(\mathrm{n}, \gamma)$ & AMS & ANL, ANU & in progress \\
\hline $124,126,132,{ }_{134} \mathrm{Xe}(\mathrm{n}, \gamma)$ & $\gamma$ spec. & Goethe U & in progress \\
\hline $136,138,140,142 \mathrm{Ce}(\mathrm{n}, \gamma)$ & $\gamma$ spec. & - & prelim,this conf. \\
\hline${ }^{147} \operatorname{Pm}(n, \gamma)$ & $\gamma$ spec. & ILL, PSI, U Seville & prelim, this conf. \\
\hline${ }^{171} \operatorname{Tm}(\mathrm{n}, \gamma)$ & $\gamma$ spec. & ILL, PSI, U Seville & prelim,this conf. \\
\hline${ }^{208} \mathrm{~Pb}(\mathrm{n}, \gamma)$ & $\beta, \gamma$ spec. & U Seville & in preparation \\
\hline${ }^{209} \operatorname{Bi}(\mathrm{n}, \gamma)$ & $\alpha, \beta, \gamma$ spec. & JRC, Geel & in preparation \\
\hline
\end{tabular}


We describe and give here preliminary results of the ${ }^{38} \operatorname{Ar}(\mathrm{n}, \gamma)^{39} \mathrm{Ar}$ experiments at stellar energies, where reaction products are counted by accelerator mass spectrometry (AMS) [5], and of ${ }^{\text {nat }} \mathrm{Kr}$ neutron activation, where ${ }^{81} \mathrm{Kr}$ and ${ }^{85 g} \mathrm{Kr}$ are counted by atom-trap trap analysis (ATTA) [6]. The ${ }^{\text {nat }} \mathrm{Ce}(\mathrm{n}, \gamma)$ reactions were revisited and $30-\mathrm{keV}$ neutron activation of radioactive targets ${ }^{147} \mathrm{Pm}$ and ${ }^{171} \mathrm{Tm}$ were performed; preliminary results are presented.

\section{2. ${ }^{36,38} \operatorname{Ar}(\mathrm{n}, \gamma)$ reactions}

Argon in the Solar System has two major isotopes ${ }^{36} \mathrm{Ar}(84.59 \%),{ }^{38} \mathrm{Ar}(15.38 \%)$ and the trace isotope ${ }^{40} \mathrm{Ar}(0.02 \%)$. This contrasts with the terrestrial (and rocky planets) abundances in reverse order ${ }^{36} \mathrm{Ar}(0.34 \%),{ }^{38} \mathrm{Ar}(0.063 \%)$ and ${ }^{40} \mathrm{Ar}(99.60 \%)$, due to radiogenic ${ }^{40} \mathrm{Ar}$ from ${ }^{40} \mathrm{~K}$
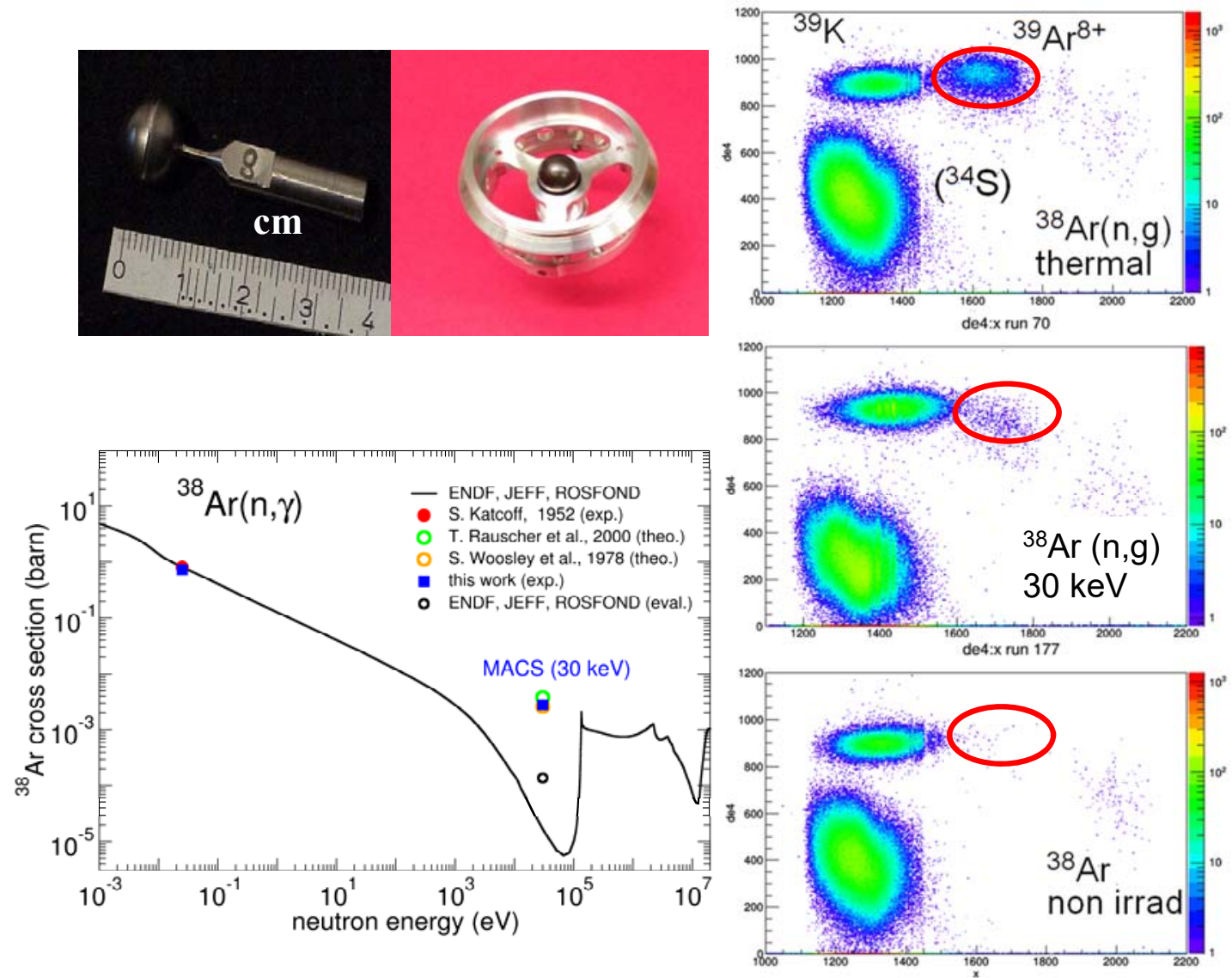

Figure 1: (top left) Ti sphere for irradiation of the ${ }^{36,38} \mathrm{Ar}$ gas targets at SARAF-LiLiT; (right) spectra of energy loss vs. focal plane position in the Enge gas-filled magnetic spectrograph. The ${ }^{39} \mathrm{Ar}$ group is separated from beam contaminants and counted; (bottom left) preliminary results of experimental and theoretical or evaluated ${ }^{38} \operatorname{Ar}(\mathrm{n}, \gamma)^{39} \mathrm{Ar}$ cross sections. The thermal cross section measured in this work is in good agreement with a previous measurement; the $30-\mathrm{keV}$ cross section is measured for the first time.

decay. The two abundant Ar isotopes, ${ }^{36} \mathrm{Ar}$ and ${ }^{38} \mathrm{Ar}$, are calculated to be produced in massive stars by oxygen burning, both hydrostatic and explosive, while primordial ${ }^{40} \mathrm{Ar}(0.02 \%)$ is 
produced by the weak s-process during He-, C- and Ne-burning [7]. A number of other neutronrich isotopes in the vicinity of Ar are not efficiently produced by charged-particle reactions and their origin is still a matter of debate [8]. Prominent examples of these include ${ }^{36} \mathrm{~S}$ and ${ }^{46,48} \mathrm{Ca}$. A promising solution is that they are produced by the weak s-process in massive stars, known to produce most of the s-nuclei between $\mathrm{Fe}$ and $\mathrm{Sr}$ during core He- and shell C-burning [8]. The sprocess flow through ${ }^{36} \mathrm{Ar},{ }^{38} \mathrm{Ar}$ and the branching point at ${ }^{39} \mathrm{Ar}$ affects the production of s-process nuclei in the Ar region, including the important cosmo/geo-chronometers ${ }^{40} \mathrm{~K}$ and ${ }^{41} \mathrm{Ca}$.

Isotopically enriched (99.9\%) ${ }^{38} \mathrm{Ar}$ gas, encapsulated in a small sphere (Fig. 1) made of titanium (diameter $=10 \mathrm{~mm}$, shell thickness $0.2 \mathrm{~mm}$ [9]), was irradiated at SARAF-LiLiT when. A thermal-neutron activation was also performed at the Soreq NRC reactor with a sphere filled with mixed ${ }^{38} \mathrm{Ar}+{ }^{\text {nat }} \mathrm{Ar}(11 \mathrm{at} \%)$ gas for internal normalization of the thermal-neutron fluence via the ${ }^{40} \operatorname{Ar}(\mathrm{n}, \gamma)^{41} \operatorname{Ar}(109 \mathrm{~min})$ reaction $\left(\sigma_{\mathrm{th}}=0.66 \mathrm{~b}[10]\right)$. In both cases, an external Au neutron monitor was placed onto the Ar sphere. The neutron-capture reaction cross section $\sigma$ of ${ }^{38} \mathrm{Ar}$ is determined from the isotopic ratio $r={ }^{39} \mathrm{Ar} /{ }^{38} \mathrm{Ar}$, measured by AMS [11], using the relation $\sigma=r / \phi t$, where $\phi t$ is the neutron fluence during the activation determined by an appropriate monitor (see [12]). Detection of ${ }^{39} \mathrm{Ar}(269 \mathrm{y})$ was performed at the ATLAS facility (Argonne National Laboratory). Positive ${ }^{38,39} \mathrm{Ar}^{8+}$ ions produced in the Electron Cyclotron Resonance ion source are accelerated through ATLAS to $6 \mathrm{MeV} / u$. The ${ }^{39} \mathrm{Ar}^{8+}$ beam is then analyzed and counted in the Enge gas-filled magnetic spectrometer to separate contaminant ions ${ }^{39} \mathrm{~K}^{8+},{ }^{34} \mathrm{~S}^{7+}$ (Fig. 1). Stable ${ }^{38} \mathrm{Ar}^{8+}$ charge is alternatively measured with a suppressed Faraday cup to extract the isotopic ratio $r$. Preliminary results for the ${ }^{38} \operatorname{Ar}(\mathrm{n}, \gamma)$ cross sections are shown in Fig.1. Irradiations of ${ }^{36} \mathrm{Ar}$ at thermal and $30-\mathrm{keV}$ neutron energy were performed in a similar way and analysis by AMS and low- level counting (LLC) are under way.

Figure 2: Detection of neutronactivated products from a ${ }^{\text {nat }} \mathrm{Kr}$-filled sphere irradiated with the SARAFLiLiT setup at $1.92 \mathrm{MeV}$ (above neutron threshold) by (top) $\gamma$ spectrometry and (bottom) atom-trap trace analysis (ATTA). The spectrum shown was measured for atmospheric $\mathrm{Kr}$ (cosmogenic ${ }^{81} \mathrm{Kr}$ and anthropogenic ${ }^{85} \mathrm{Kr}$ ). The ${ }^{81,85} \mathrm{Kr}$ signals for the (n, $\left.\gamma\right)$ irradiated samples are ${ }^{81} \mathrm{Kr} / \mathrm{Kr}=$ $1.56 \times 10^{-12},{ }^{85 \mathrm{~g}} \mathrm{Kr} / \mathrm{Kr}=1.77 \times 10^{-12}$.
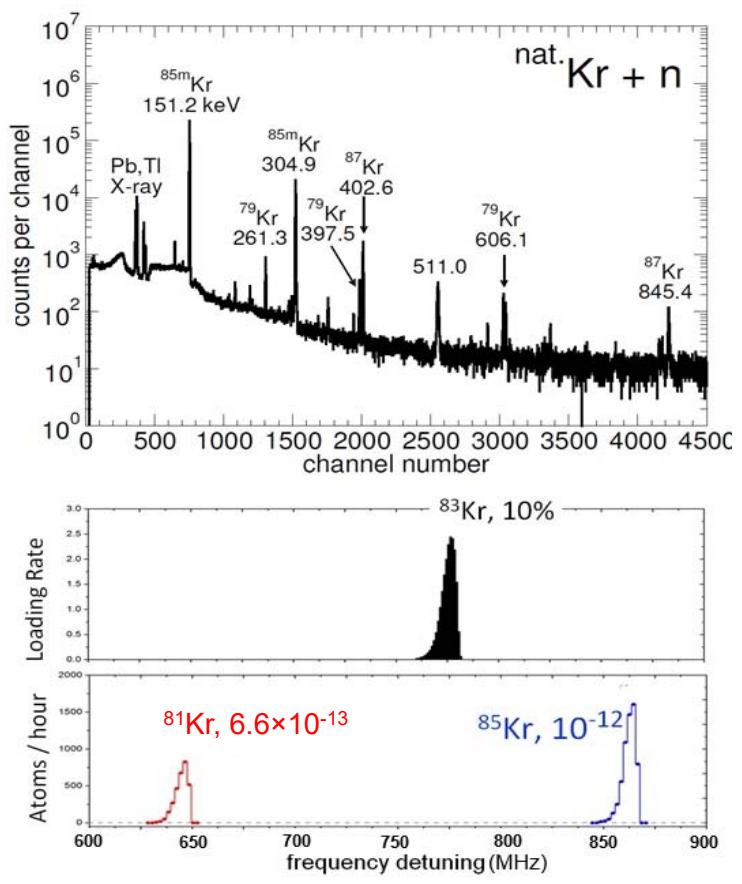


\section{3. ${ }^{78,80,84,86} \operatorname{Kr}(\mathrm{n}, \gamma)$ and ${ }^{80,82,86} \mathrm{Kr}(\gamma, \mathrm{n})$ reactions}

The abundance of natural $\mathrm{Kr}$ isotopes, situated in the table of nuclides near the end of the socalled "weak" s-process [13], is considered an important test for our understanding of the s-process evolution. The importance of the ${ }^{85} \mathrm{Kr}$ branching point in the vicinity of the neutron-magic nuclide ${ }^{86} \mathrm{Kr}$ has been stressed [14]. We performed neutron activations of ${ }^{\text {nat }} \mathrm{Kr}$ gas samples with a setup similar to that described above. Short-lived ${ }^{77,79,85 \mathrm{~m}, 87} \mathrm{Kr}$ products were counted by $\gamma$ spectrometry while long-lived ${ }^{81} \operatorname{Kr}\left(2.3 \times 10^{5}\right.$ y) and ${ }^{859} \operatorname{Kr}(10.8$ y) are detected by LLC and by atom-trap trace analysis (ATTA) [6] where individual atoms trapped in a magneto-optical trap are counted via repeated decay and excitation of their atomic transition (Fig. 2). A separate activation was performed with a proton beam incident on LiLiT below the neutron threshold to measure the ${ }^{78,80,82,86} \operatorname{Kr}(\gamma, \mathrm{n})$ cross sections. The results are being analyzed to extract MACS values.

\section{4. $\quad 136,138,140,142 \mathrm{Ce}(\mathrm{n}, \gamma)$ and ${ }^{140} \mathrm{Ce}(\gamma, \mathrm{n}){ }^{139} \mathrm{Ce}$ reactions}

The disentanglement of the different heavy-nuclide synthesis modes ( $s-, r$ - and $p$ processes) requires reliable and precise stellar neutron-capture cross sections. Such is the case for the Ce isotopes, studied in [15]. In particular, ${ }^{140} \mathrm{Ce}$ is found to be one of the most important nuclides in the network of s-process reactions, affecting the abundances of a large number of isotopes [16]. First results of ${ }^{136,138,140,142} \mathrm{Ce}(\mathrm{n}, \gamma)$ cross sections measured at SARAF-LiLiT were presented [17]. We correct here one of these cross section measured values, namely ${ }^{138} \mathrm{Ce}(\mathrm{n}, \gamma){ }^{139} \mathrm{Ce}$ for which the ${ }^{139} \mathrm{Ce}$ activation product was observed to have a significant contribution from the ${ }^{140} \mathrm{Ce}(\gamma, \mathrm{n}){ }^{139} \mathrm{Ce}$ due, in part, to the relatively small and large natural abundances of ${ }^{138} \mathrm{Ce}$ and ${ }^{140} \mathrm{Ce}$, respectively. In order to measure the $(\gamma, \mathrm{n})$ cross section, we irradiated a metallic ${ }^{\text {nat }} \mathrm{Ce}$ target ( $25 \mathrm{~mm}$ diameter, $640 \mu \mathrm{m}$ thickness), prepared similarly as in [17] with $\gamma$-rays generated by the LiLiT setup when bombarded by an intense ( 1-1.5 mA) proton beam at an energy of $1.81 \mathrm{MeV}$ (below the ${ }^{7} \mathrm{Li}(\mathrm{p}, \mathrm{n})$ threshold). Figure 3 shows $\gamma$-spectra for the Ce target activated above and below neutron threshold. Quantitative subtraction of the ${ }^{139} \mathrm{Ce}$ production by $(\gamma, \mathrm{n})$ from that of $(\mathrm{n}, \gamma)$ allowed us to extract the $(\mathrm{n}, \gamma)$ cross section, although with increased uncertainty. Table 2 lists preliminary results of the ${ }^{136,138,140,142} \mathrm{Ce}(\mathrm{n}, \gamma)$ MACS, superseding those presented in [17].

Table 2: Preliminary neutron-capture MACS (30 keV) for ${ }^{136,138,140,142} \mathrm{Ce}$

\begin{tabular}{ccc}
\hline \hline Target & $\begin{array}{c}\text { MACS-30 keV } \\
\text { this work } \\
(\mathrm{mb})\end{array}$ & $\begin{array}{c}\text { MACS-30 keV } \\
\text { KADONIS [18] } \\
(\mathrm{mb})\end{array}$ \\
\hline${ }^{136} \mathrm{Ce} \rightarrow{ }^{137 \mathrm{~m}} \mathrm{Ce}$ & in progress & $328 \pm 21$ \\
${ }^{136} \mathrm{Ce} \rightarrow{ }^{137 \mathrm{~g}} \mathrm{Ce}$ & in progress & $28.2 \pm 1.6$ \\
${ }^{138} \mathrm{Ce} \rightarrow{ }^{139} \mathrm{Ce}$ & $175.8 \pm 9.0$ & $179 \pm 5$ \\
${ }^{140} \mathrm{Ce} \rightarrow{ }^{141} \mathrm{Ce}$ & $10.9 \pm 0.3$ & $11.0 \pm 0.4$ \\
${ }^{142} \mathrm{Ce} \rightarrow{ }^{143} \mathrm{Ce}$ & $26.2 \pm 0.5$ & $28 \pm 1$ \\
\hline \hline
\end{tabular}



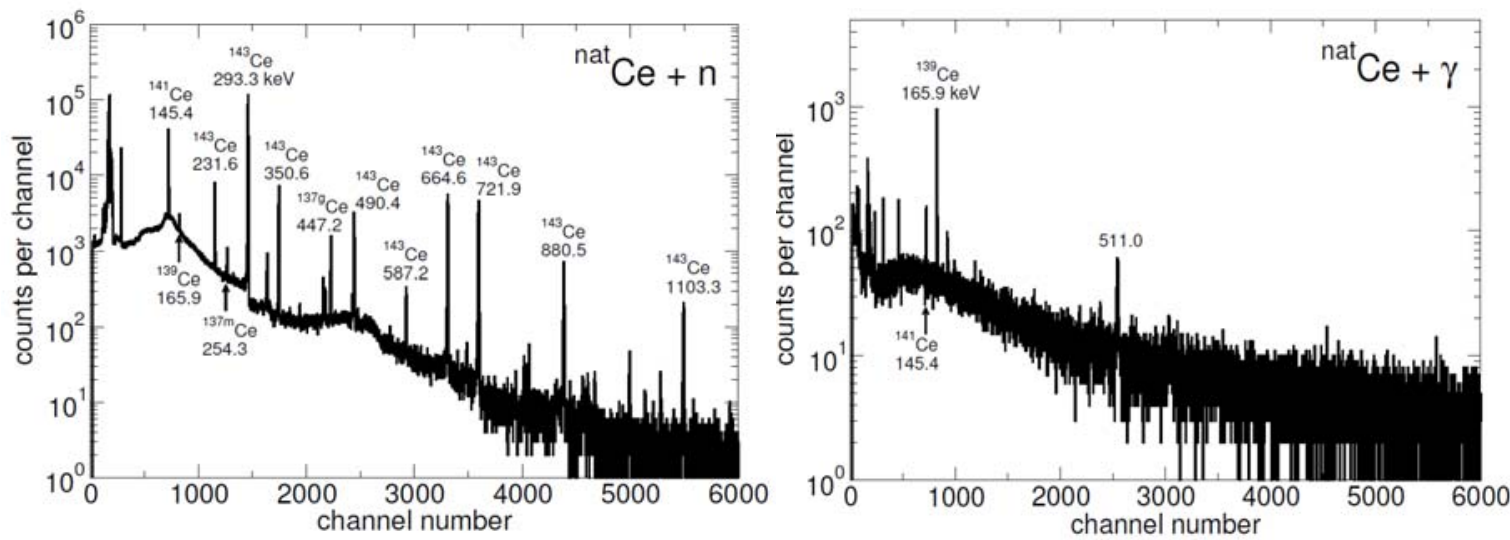

Figure 3: $\gamma$-spectra measured with a nat Ce sample activated by (left) 30-keV neutrons from LiLiT with a proton beam of $1.92 \mathrm{MeV}$; (right) $17.6,14.6 \mathrm{MeV} \gamma$-rays from LiLiT with a proton beam of $1.81 \mathrm{MeV}$ (under the ${ }^{7} \mathrm{Li}(\mathrm{p}, \mathrm{n})$ neutron threshold of $1.880 \mathrm{MeV}$ ).

\section{5. $\quad{ }^{147} \mathrm{Pm}(\mathrm{n}, \gamma)$ and ${ }^{171} \mathrm{Tm}(\mathrm{n}, \gamma)$ reactions}

Branching-point nuclides play an important role in the evolution of the stellar s-process along and near the valley of stability: these are $\beta$-unstable nuclides whose stellar life time is long in comparison (or at least comparable) with the mean time for neutron capture and the competition between decay and neutron capture determines the s-process path. This mean time, determined by the MACS and the neutron density of the stellar site, is generally much less known experimentally for unstable nuclides than for stable nuclides, much due to the difficulty of producing the adequate targets necessary for cross section measurement. We report here on recent measurements performed on ${ }^{147} \mathrm{Pm}$ and ${ }^{171} \mathrm{Tm}$, two important branching points of the main s-process. The element Pm has no stable isotopes and the two main nuclides responsible for the continuity of the s-process evolution are ${ }^{147} \mathrm{Pm}(2.62 \mathrm{y})$ and ${ }^{149} \mathrm{Pm}(53.1 \mathrm{~h})$. The ${ }^{171} \mathrm{Tm}(1.92 \mathrm{y})$ nuclide operates as a branching point, removed by two neutrons from the valley of stability.

${ }^{147} \mathrm{Pm}$ and ${ }^{171} \mathrm{Tm}$ targets were prepared for experiments at nTOF [19] and subsequently shipped to SARAF for activation at LiLiT. The isotopes ${ }^{147} \mathrm{Pm},{ }^{171} \mathrm{Tm}$ were produced by neutron irradiation at the high flux reactor at Institut Laue-Langevin (Grenoble) of pellets of ${ }^{146} \mathrm{Nd}_{2} \mathrm{O}_{3}$ (98.2 mg) enriched to $98.8 \%$ and ${ }^{170} \mathrm{Er}_{2} \mathrm{O}_{3}(238 \mathrm{mg}$ ) enriched to $98.1 \%$, respectively. The pellets were encapsulated into high purity quartz ampules, then irradiated at ILL for a period of 54 days with an average neutron flux of $8.2 \times 10^{14} \mathrm{n} / \mathrm{cm}^{2} / \mathrm{s}$. After a cooling period of approximately 1.5 years, the samples were shipped to PSI where they underwent chemical processing. Pm and Tm extracted from the pellets were chemically purified prior to making them suitable targets. The material was then electroplated onto $5 \mu \mathrm{m}$ thick aluminum backings resulting into two high quality targets of $22 \mathrm{~mm}$ diameter with a total of $3.5 \mathrm{mg}$ of ${ }^{171} \mathrm{Tm}(140 \mathrm{GBq})$ and $72 \mu \mathrm{g}$ of ${ }^{147} \mathrm{Pm}(2.5$ $\mathrm{GBq}$ ) [20]. For handling and shipping, the radioactive targets were sealed in air-tight $\mathrm{Al}$ containers (Fig. 4), used also for the neutron irradiation at SARAF-LiLiT. We present below preliminary results of the $30-\mathrm{keV}$ neutron activation of the ${ }^{171} \mathrm{Tm},{ }^{147} \mathrm{Pm}$ targets, performed in Spring 2016. $\mathrm{An} \mathrm{Au}$ foil affixed onto the Al container was used to monitor the neutron fluence; irradiation of an $\mathrm{Au}$ foil of the same size as the ${ }^{171} \mathrm{Tm}$ target mounted in a dummy $\mathrm{Al}$ container was performed 

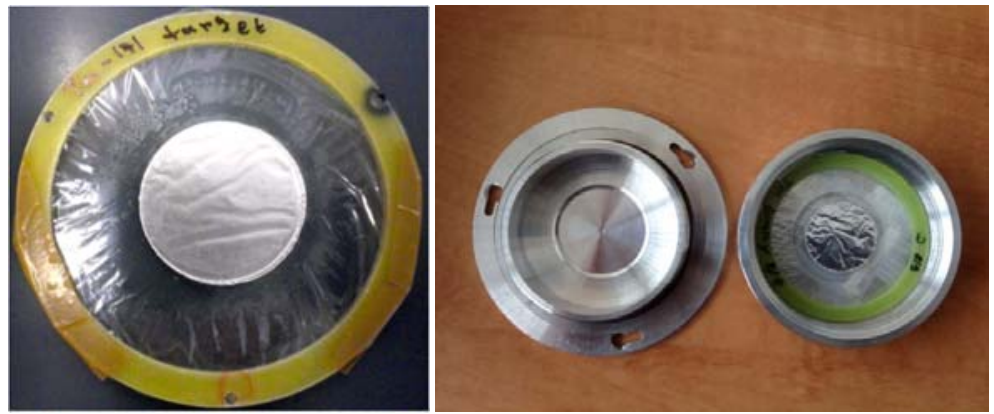

Fig. 4: (left) Target mount as prepared at PSI [20] for ${ }^{147} \mathrm{Pm}$ and ${ }^{171} \mathrm{Tm}$. The material is deposited on a A1 backing (22 $\mathrm{mm}$ diameter) and sealed by Mylar foils mounted on a plastic ring; (right) for shipping and irradiating at SARAF, each target was sealed in an Al container made of two parts. The Al thickness seen by incident neutrons is $1 \mathrm{~mm}$.

to benchmark a detailed simulation of the irradiation setup. The activation of the ${ }^{171} \mathrm{Tm}(\mathrm{Au}$ dummy) target lasted $\sim 8$ hours ( $\sim 0.8$ hours) at an average proton beam intensity of $\sim 1.1 \mathrm{~mA}(\sim 0.4$ $\mathrm{mA})$. After irradiation, the ${ }^{171} \mathrm{Tm}$ target was positioned at a distance of $92 \mathrm{~mm}$ from a shielded $\mathrm{Ge}$ detector, using three $\mathrm{Pb}$ plates $(2 \mathrm{~mm}$ thick each) and one $\mathrm{Cu}(2 \mathrm{~mm})$ used as absorbers. The plates significantly absorb background photons and the low-energy $67-\mathrm{keV} \gamma$ 's from ${ }^{171} \mathrm{Tm}$ decay while the higher-energy $\gamma$ 's (1094 (6\%), 1387 (5.6\%), 1466 (4.5\%), $1529(5.1 \%)$ and $1608(4.1 \%))$ from ${ }^{172} \mathrm{Tm}$ decay are transmitted more efficiently (see Fig. 5 left).
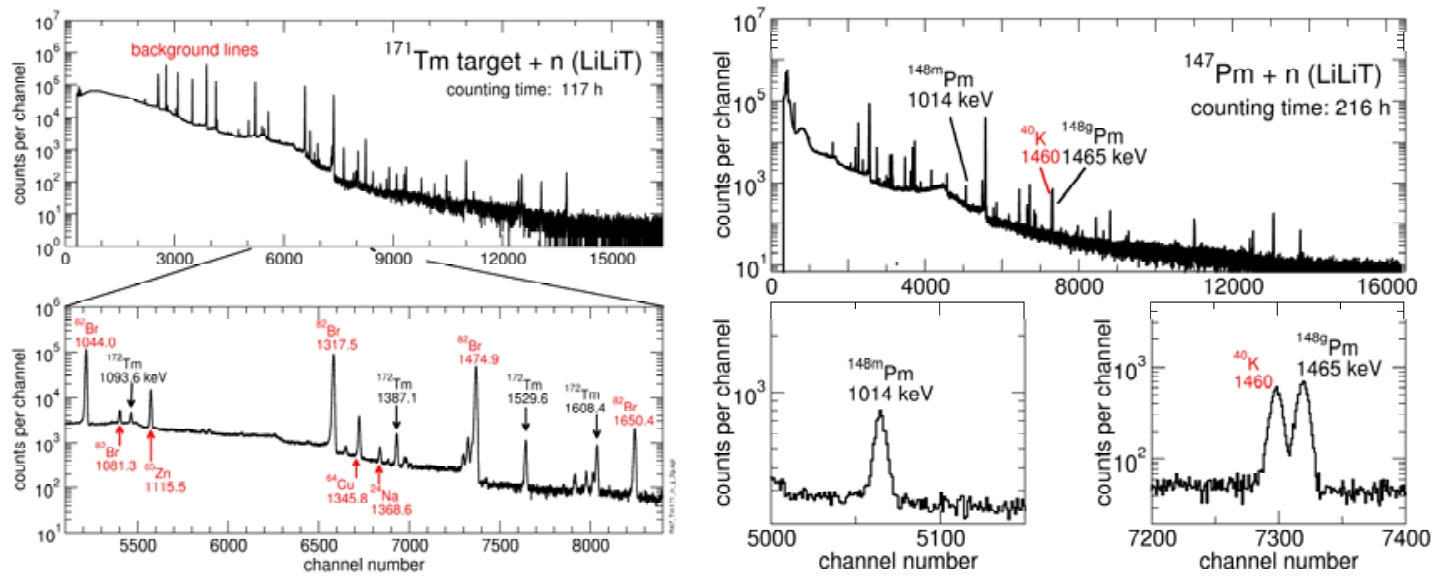

Figure 5: (left) $\gamma$ spectrum (integrated over the whole timed series, see text) for the activated ${ }^{171} \mathrm{Tm}$ target at SARAF-LiLiT; (right) Same for activated ${ }^{147} \mathrm{Pm}$ target. The ground state and isomeric state population are observed. Identified activation background lines are indicated (red).

Counting of the ${ }^{172} \mathrm{Tm}$ decay lines were made as a timed series of $10800 \mathrm{sec}$ long counting periods between 13.3 and 145 hours after end of activation. The decay curves measured for the four $\gamma$ lines listed above are consistent with the half-life of ${ }^{172} \mathrm{Tm}(63.6 \mathrm{~h})$. The photopeak efficiency calibration of the Ge detector with the absorber and shielding configuration described above was determined empirically by placing a calibrated ${ }^{152} \mathrm{Eu}$ point source in the dummy $\mathrm{Al}$ holder in conditions (target holder, distance and absorber setup) identical to those of the ${ }^{171} \mathrm{Tm}$ target. Summing and target size effects are taken into consideration by a detailed simulation. The 
preliminary value we extract for the ${ }^{171} \mathrm{Tm}(\mathrm{n}, \gamma){ }^{172} \mathrm{Tm}$ cross section (averaged over the experimental neutron spectrum) is $170(18) \mathrm{mb}$, corresponding to a MACS (30 keV) value of 198(22) $\mathrm{mb}$, significantly lower than the previous experimental value MACS $(25 \mathrm{keV})=350(30) \mathrm{mb}$ [21].

The ${ }^{147} \mathrm{Pm}$ target was activated in similar conditions as ${ }^{171} \mathrm{Tm}$ and owing to a lower activity was counted at a distance of $52 \mathrm{~mm}$ from the Ge detector with one 2-mm $\mathrm{Pb}$ and one 2-mm Cu absorber. A $\gamma$ spectrum is illustrated in Fig. 5 (right), showing lines of the ground state ${ }^{148 g} \mathrm{Pm}$ $(1465 \mathrm{keV})$ and isomeric ${ }^{148 \mathrm{~m}} \mathrm{Pm}(1014 \mathrm{keV})$ decays.

This work was supported by Pazi Foundation (Israel), Israel Science Foundation (Grant 1387/15) and the US Department of Energy, Office of Nuclear Physics, under Contract NoDEAC02-06CH11357. D.S.G. acknowledges the support by the U.S. Department of Energy, Office of Nuclear Physics, under grant No. DE-FG02-96ER40978. This research has received funding from the European Research Council under the European Unions's Seventh Framework Program (FP/2007-2013) /ERC Grant Agreement n. 615126.

\section{References}

[1] S. Halfon et al., Rev. Sci. Instrum. 84 (2013) 123507.

[2] S. Halfon et al., Rev. Sci. Instrum., 85 (2014) 056105.

[3] M. Tessler et al., Phys. Lett. B 751 (2015) 418

[4] S. Pavetich et al., Proc. of Deutsche Physikalische Gesellshaft Spring Meeting, Heidelberg, 23-27 March, 2015.

[5] W. Kutschera, Adv. in Physics 1 (2016) 570

[6] C. Y. Chen, Science 286, 1139 (1999)

[7] R.D. Hoffman et al., Astrophys. J. 521, 735 (1999)

[8] R. Reifarth, K. Schwarz and F. Kaeppeler, Astrophys. J. 528, 573 (2000)

[9] G. Rupp et al., Nucl. Instr. Meth, A608, 152 (2009)

[10] S. F. Mughabghab, Atlas of Neutron Resonances, Elsevier, 2006.

[11] P. Collon et al., Nucl. Instr. Meth. Phys. Res. B 223-224, 428 (2004)

[12] H. Nassar et al., Phys. Rev. Lett. 94, 092504 (2005)

[13] F. Kaeppeler et al., Rev. Mod. Phys. 83, 157 (2011)

[14] R. Raut et al., Phys. Rev. Lett. 111, 112501 (2013)

[15] F. Kaeppeler, et al., Phys. Rev. C 53, 1397 (1996)

[16] A. Koloczek et al., Atomic Data and Nuclear Data Tables 108, 1 (2016)

[17] M. Paul et al., PoS (NIC XIII) 059 (2014)

[18] KADONIS, http://www.kadonis.org/

[19] C. Guerrero et al., PoS (NIC XIV), to be published.

[20] S. Heinitz, Radiochim. Acta, accepted for publication.

[21] R. Reifarth et al., Nuclear Physics A718, 478c (2003) 